\title{
PERIODIC SOLUTIONS OF A MODEL OF LOTKA-VOLTERRA WITH VARIABLE STRUCTURE AND IMPULSES
}

\author{
Katya Dishlieva ${ }^{1}$, Andrey Antonov ${ }^{2}$, Angel Dishliev ${ }^{3}$ \\ ${ }^{1}$ Department of Diferential Equations, Faculty of Applied Mathematics and Informatics, \\ Technical University of Sofia, Sofia, Bulgaria \\ ${ }^{2}$ Department of Mathematics, University of Chemical Technology and Metallurgy, \\ Sofia, Bulgaria \\ ${ }^{2}$ Department of Mathematics, University of Chemical Technology and Metallurgy, \\ Sofia, Bulgaria
}

\begin{abstract}
We consider a generalized version of the classical Lotka Volterra model with differential equations. The version has a variable structure (discontinuous right hand side) and the solutions are subjected to the discrete impulsive effects. The moments of right hand side discontinuity and the moments of impulsive effects coincide and they are specific for each solution. Using the Brouwer fixed point theorem, sufficient conditions for the existence of periodic solution are found.
\end{abstract}

\section{Keywords}

variable structure; impulses; periodic solutions

\section{Academic Discipline And Sub-Disciplines}

Provide examples of relevant academic disciplines for this journal: E.g., History; Education; Sociology; Psychology; Cultural Studies;

AMS (MOS) Subject Classification: 34A37, 34C25

\section{Council for Innovative Research}

Peer Review Research Publishing System

\section{Journal: JOURNAL OF ADVANCES IN MATHEMATICS}

Vol.11, No.6

www.cirjam.com, editorjam@gmail.com 


\section{INTRODUCTION}

We assume that the predator-prey community is subjected to the external effects. These effects are discrete in time and relate to:

1. Impulsive changes in the biomass amount of both species (forming part of the community). Mathematically this is expressed through a jump-like change of the state (phase or solution) of the modeling system;

2. Instantaneous rate of change of community development speed. Usually this effect is associated with a jump-like change system parameters. Generally, this effect is expressed by changing the structure of the modeling.

We shall assume that these jump-like changes (both the state and also the speed of community development) are performed simultaneously. The moments of external effects are variable and depend on the quantities of biomass, i.e. on the specific solution of modeling system. The above-described community has different variations of mathematical modeling. In general, these options depend on:

1. The nature of interactions between the species in the periods outside of the moments of external influences (reflected in the type of right hand side of modeling system);

2. Impulsive moments and the way they are set (these are the moments that the external effects take place);

3. Impulsive effects (the quantities of withdrawal or adding biomass to the predator and prey);

4. Changing the structure of the modeling system (values which change the parameters of the community development), etc.

Impulsive differential equations are suitable mathematical tool for processes modeling with short-term changes in their phase state. This type of equations is developed intensively because of their serious applications. Concrete results relating to the Lotka-Volterra models, subjected to pulse effects, are contained in [5], [6], [9], [10] and [11].

Various aspects of the qualitative theory of impulsive differential equations with variable structure and impulses are discussed in [1], [2], [4], [7] and [8].

This paper contains the application of the above mentioned type of differential equations. More precisely, conditions associated with the elements of system, ensuring the existence of periodic solutions are found.

\section{DESCRIPTION OF THE MODEL}

Consider the following initial value problem for systems of differential equations with variable structure and moments of impulsive effects

$$
\begin{aligned}
& \frac{d m}{d t}=f_{i}^{1}(m, M)=m\left(r_{i}^{1}-q_{i}^{1} M\right),(m(t), M(t)) \notin \Phi_{i} \Leftrightarrow t_{i-1}<t<t_{i} ; \\
& \frac{d M}{d t}=f_{i}^{2}(m, M)=-M\left(r_{i}^{2}-q_{i}^{2} m\right),(m(t), M(t)) \notin \Phi_{i} \Leftrightarrow t_{i-1}<t<t_{i} \\
& m\left(t_{i}+0\right)=g_{i}\left(m\left(t_{i}\right)\right),\left(m\left(t_{i}\right), M\left(t_{i}\right)\right) \in \Phi_{i} ; \\
& M\left(t_{i}+0\right)=M_{i+1}^{00},\left(m\left(t_{i}\right), M\left(t_{i}\right)\right) \in \Phi_{i}, \quad i=1,2, \ldots ; \\
& m(0)=m_{0}, \quad M(0)=M_{0},
\end{aligned}
$$

where:

- $m=m(t)>0$ and $M=M(t)>0$ are the prey and predator biomasses at the moment $t \geq 0$;

- $r_{i}^{1}=$ const $>0$ and $r_{i}^{2}=$ const $>0$ are specific growth factors, relevant to the first species (prey) and second (predator), respectively;

- $q_{i}^{1}=$ const $>0$ and $q_{i}^{2}=$ const $>0$ are the coefficients indicating interspecies competition. In the common case, they are different for the prey and predator;

- $\left(m_{i}^{00}, M_{i}^{00}\right)=\left(r_{i}^{2} / q_{i}^{2}, r_{i}^{1} / q_{i}^{1}\right)$; 
- $\Phi_{1}, \Phi_{2}, \ldots$ are switching sets, $\Phi_{i}=\left\{(m, M) ; m>m_{i}^{00}, M=M_{i}^{00}\right\}, i=1,2, \ldots$;

- $g_{i}: R^{+} \rightarrow R^{+}$

$-t_{1}, t_{2}, \ldots$ are switching moments;

- $m_{0}>0$ and $M_{0}>0$ are the prey and predator biomasses at the initial moment $t=0$.

Remark 1. The solution $\left(m\left(t ; m_{0}, M_{0}\right), M\left(t ; m_{0}, M_{0}\right)\right)$ of the initial value problem (1)-(5) is defined as follows:

1.1. For $0=t_{0} \leq t<t_{1}$ :

1.1.1. $\left(m\left(t ; m_{0}, M_{0}\right), M\left(t ; m_{0}, M_{0}\right)\right)$ coincides with the solution of the system (1), (2) for $i=1$ with initial condition (5);

1.1.2. $\left(m\left(t ; m_{0}, M_{0}\right), M\left(t ; m_{0}, M_{0}\right)\right) \notin \Phi_{1} ;$

1.2. For $t=t_{1}$ :

1.2.1. $\left(m\left(t_{1} ; m_{0}, M_{0}\right), M\left(t_{1} ; m_{0}, M_{0}\right)\right) \in \Phi_{1} ;$

1.2.2. $m\left(t_{1}+0 ; m_{0}, M_{0}\right)=g_{1}\left(m\left(t_{1} ; m_{0}, M_{0}\right)\right)=m_{1}^{+}, M\left(t_{1}+0 ; m_{0}, M_{0}\right)=M_{2}^{00}=M_{1}^{+} ;$

2.1. For $t_{1}<t<t_{2}$ :

2.1.1. $\left(m\left(t ; m_{0}, M_{0}\right), M\left(t ; m_{0}, M_{0}\right)\right)$ coincides with the solution of system (1), (2) for $i=2$ with initial condition $m\left(t_{1}\right)=m_{1}^{+}, M\left(t_{1}\right)=M_{1}^{+}$;

2.1.2. $\left(m\left(t ; m_{0}, M_{0}\right), M\left(t ; m_{0}, M_{0}\right)\right) \notin \Phi_{2}$;

2.2. For $t=t_{2}$ :

2.2.1. $\left(m\left(t_{2} ; m_{0}, M_{0}\right), M\left(t_{2} ; m_{0}, M_{0}\right)\right) \in \Phi_{2}$;

2.2.2. $m\left(t_{2}+0 ; m_{0}, M_{0}\right)=g_{2}\left(m\left(t_{2} ; m_{0}, M_{0}\right)\right)=m_{2}^{+}, M\left(t_{2}+0 ; m_{0}, M_{0}\right)=M_{3}^{00}=M_{2}^{+}$;

and so on.

Further, we use the notations $m_{i}^{+}=g_{i}\left(m\left(t_{i} ; m_{0}, M_{0}\right)\right), M_{i}^{+}=M_{i+1}^{00}, \quad i=1,2, \ldots$.

For $i=1,2, \ldots$, consider the corresponding initial value problems

$$
\begin{aligned}
& \frac{d m}{d t}=f_{i}^{1}(m, M) ; \\
& \frac{d m}{d t}=f_{i}^{1}(m, M) ; \\
& m\left(t_{0 i}\right)=m_{0 i}, M\left(t_{0 i}\right)=M_{0 i} .
\end{aligned}
$$

Denote their solutions by $\left(m_{i}\left(t ; t_{0 i} ; m_{0 i}, M_{0 i}\right), M_{i}\left(t ; t_{0 i} ; m_{0 i}, M_{0 i}\right)\right)$, where the initial points $\left(t_{0 i}, m_{0 i}, M_{0 i}\right)$ $\in R^{+} \times R^{+} \times R^{+}, i=1,2, \ldots$.

Remark 2. It is known that for $i=1,2, \ldots$, the system (6), (7) (without impulses) has the following properties: 
1. Unstable (saddle) stationary point $(0,0)$. It is filled $f_{i}^{1}(0,0)=f_{i}^{2}(0,0)=0$;

2. Stable stationary point $\left(m_{i}^{00}, M_{i}^{00}\right)=\left(r_{i}^{2} / q_{i}^{2}, r_{i}^{1} / q_{i}^{1}\right)$. It is filled $f_{i}^{1}\left(m_{i}^{00}, M_{i}^{00}\right)=f_{i}^{2}\left(m_{i}^{00}, M_{i}^{00}\right)=0$;

3. First integral of the form

$V_{i}(m, M)=q_{i}^{1} M+q_{i}^{2} m-r_{i}^{1} \ln M-r_{i}^{2} \ln m+r_{i}^{1}\left(\ln ^{r_{i}^{1}} / q_{i}^{1}-1\right)+r_{i}^{2}\left(\ln ^{r_{i}^{2}} / q_{i}^{2}-1\right)$

4. For each constant $c \geq 0$, implicitly given curve

$\gamma_{i}^{c}=\left\{(m, M) ; V_{i}(m, M)=c\right\}$

is a trajectory of system (1), (2). This trajectory is closed;

5. For each constant $c \geq 0$, the set

$G_{i}^{c}=\left\{(m, M) ; V_{i}(m, M) \leq c\right\}$

is a connected domain, situated in $R^{+} \times R^{+}$, which possesses contour $\partial G_{i}^{c}=\gamma_{i}^{c}$;

6. If $0<c_{1}<c_{2}$, then $\gamma_{i}^{c_{1}} \subset G_{i}^{c_{2}}$;

7. Let $c$ and $C$ be arbitrary constants such that $0<c<C$. We assume that for $i=1,2, \ldots$, the phase space of system (1), (2) is defined as $G_{i}^{c, C}=\overline{G_{i}^{C} \backslash G_{i}^{c}}$, i.e.

$G_{i}^{c, C}=\left\{(m, M) ; c \leq V_{i}(m, M) \leq C\right\}$;

8. The switching sets of system (1)-(4) satisfy the inclusions $\Phi_{i} \subset G_{i}^{c, C}$. It is true $\Phi_{i}=\Phi_{i}^{c, C}=G_{i}^{c, C} \cap\left\{(m, M) ; m>m_{i}^{00}, M=M_{i}^{00}\right\}$.

9. If the point $\left(m_{0 i}, M_{0 i}\right) \in R^{+} \times R^{+}$, then the solution $\left(m_{i}\left(t ; t_{0 i} ; m_{0 i}, M_{0 i}\right), M_{i}\left(t ; t_{0 i} ; m_{0 i}, M_{0 i}\right)\right)$ of the initial value problem (6), (7), (8) is continuable up to $\infty$;

10. If the initial point $\left(m_{0 i}, M_{0 i}\right) \in G_{i}^{c, C}$, then the solution of (6), (7), (8) for each $t \geq t_{0 i}$ also belongs to $G_{i}^{c, C}$;

11. Because the set $G_{i}^{c, C}$ is closed and bounded and the functions $f_{i}^{1}$ and $f_{i}^{2}$ are continuous on $G_{i}^{c, C}$, then there exist positive constants $M_{f_{i}^{1}}$ and $M_{f_{i}^{2}}$, such that

$\left(\forall(m, M) \in G_{i}^{c, C}\right) \Rightarrow\left|f_{i}^{1}(m, M)\right| \leq M_{f_{i}^{1}},\left|f_{i}^{2}(m, M)\right| \leq M_{f_{i}^{2}}$.

12. It is fulfilled

$\left(m\left(t ; m_{0}, M_{0}\right), M\left(t ; m_{0}, M_{0}\right)\right)$

$$
=\left\{\begin{array}{l}
\left(m_{1}\left(t ; t_{0} ; m_{0}^{+}, M_{0}^{+}\right), M_{1}\left(t ; t_{0} ; m_{0}^{+}, M_{0}^{+}\right)\right), 0=t_{0} \leq t \leq t_{1} ; \\
\left(m_{i}\left(t ; t_{i-1} ; m_{i-1}^{+}, M_{i-1}^{+}\right), M_{i}\left(t ; t_{i-1} ; m_{i-1}^{+}, M_{i-1}^{+}\right)\right), t_{i-1}<t \leq t_{i}, \quad i=2,3, \ldots,
\end{array}\right.
$$

where $\left(m_{0}^{+}, M_{0}^{+}\right)=\left(m_{0}, M_{0}\right) ;$

13. The following inequalities are valid

$$
\left(m\left(t ; m_{0}, M_{0}\right), M\left(t ; m_{0}, M_{0}\right)\right)
$$




$$
=\left(m_{i}\left(t ; t_{i-1} ; m_{i-1}^{+}, M_{i-1}^{+}\right), M_{i}\left(t ; t_{i-1} ; m_{i-1}^{+}, M_{i-1}^{+}\right)\right)<M_{i}^{00}, t_{i-1}<t<t_{i}, i=1,2, \ldots .
$$

Remark 3. Further, we denote by $m_{i}^{c, \max }$ and $m_{i}^{\mathrm{C}, \max }$ the bigger solutions of the equations

$$
V_{i}\left(m, M_{i}^{00}\right)=V_{i}\left(m, \frac{r_{i}^{1}}{q_{i}^{1}}\right)=c \Leftrightarrow m-\frac{r_{i}^{2}}{q_{i}^{2}} \ln m=\frac{r_{i}^{2}}{q_{i}^{2}}\left(1-\ln \frac{r_{i}^{2}}{q_{i}^{2}}\right)+\frac{c}{q_{i}^{2}}
$$

and

$V_{i}\left(m, M_{i}^{00}\right)=V_{i}\left(m, \frac{r_{i}^{1}}{q_{i}^{1}}\right)=C \Leftrightarrow m-\frac{r_{i}^{2}}{q_{i}^{2}} \ln m=\frac{r_{i}^{2}}{q_{i}^{2}}\left(1-\ln \frac{r_{i}^{2}}{q_{i}^{2}}\right)+\frac{C}{q_{i}^{2}}$,

respectively. The constants $m_{i}^{c, \text { min }}$ and $m_{i}^{\mathrm{C}, \mathrm{min}}$ are smaller solutions of the above two equations. We introduce the following sets

$\Phi_{i}^{c, C, \max }=\left\{(m, M) ; m_{i}^{c, \text { max }} \leq m \leq m_{i}^{C, \max }, \quad M=M_{i}^{00}\right\} \subset G_{i}^{c, C}, \quad i=1,2, \ldots$

and

$\Phi_{i}^{c, C, \min }=\left\{(m, M) ; m_{i}^{C, \min } \leq m \leq m_{i}^{c, \min }, \quad M=M_{i}^{00}\right\} \subset G_{i}^{c, C}, \quad i=1,2, \ldots$.

It is clear that the switching sets

$\Phi_{i}=\Phi_{i}^{c, C}=\Phi_{i}^{c, C, \max }, i=1,2, \ldots$.

Remark 4. It can be seen immediately that the sets $\Phi_{i}^{c, C, \min }$ and $\Phi_{i}^{c, C, \max }$ are closed intervals located in $R^{+} \times R^{+}$. This means that they are compact, connected and convex sets.

Remark 5. Pay attention that for $M(t)=M_{i}^{00}$ and $m(t)>m_{i}^{00}$, the right hand side of (1) becomes zero. Therefore, we have $d m / d t=0$. It can be shown that just then the victim's biomass is maximum. Consequently, the withdrawal of biomass from the victim in these moments $\left(t_{1}, t_{2}, \ldots\right)$ is justified.

\section{EXISTENCE OF PERIODIC SOLUTIONS}

Introduce the following conditions:

H1. There exists $k_{0} \in N$ such that

$r_{k_{0}+i}^{1}=r_{i}^{1} ; \quad r_{k_{0}+i}^{2}=r_{i}^{2} ; \quad q_{k_{0}+i}^{1}=q_{i}^{1} ; \quad q_{k_{0}+i}^{2}=q_{i}^{2}, \quad i=1,2, \ldots$.

H2. The functions $g_{i} \in C\left[\Phi_{i}^{c, C, \max }, R^{+}\right], \quad g_{i}\left(\Phi_{i}^{c, C, \max }\right) \subset\left\{m ; m_{i+1}^{C, \min }<m<m_{i+1}^{c, \min }\right\} \quad$ and for each $m \in \Phi_{k_{0}}^{c, C, \max }$, it is satisfied $g_{k_{0}+i}(m)=g_{i}(m), i=1,2, \ldots$.

Remark 6. From condition $\mathrm{H} 1$, we obtain the equalities:

$$
\begin{aligned}
& m_{k_{0}+i}^{00}=m_{i}^{00} ; M_{k_{0}+i}^{00}=M_{i}^{00} ; V_{k_{0}+i}(m, M)=V_{i}(m, M),(m, M) \in R^{+} \times R^{+} ; \\
& \gamma_{k_{0}+i}^{c}=\gamma_{i}^{c}, c=\text { const }>0 ; G_{k_{0}+i}^{c, C}=G_{i}^{c, C}, c=\text { const }, C=\text { const }, 0<c<C ; \\
& m_{k_{0}+i}^{c, \text { min }}=m_{i}^{c, \text { min }} ; \quad m_{k_{0}+i}^{C, \min }=m_{i}^{C, \min } ; m_{k_{0}+i}^{c, \text { max }}=m_{i}^{c, \max } ; m_{k_{0}+i}^{C, \max }=m_{i}^{C, \max } ; \\
& \Phi_{k_{0}+i}^{c, C, \min }=\Phi_{i}^{c, C, \min } ; \quad \Phi_{k_{0}+i}^{c, C, \max }=\Phi_{i}^{c, C, \max } .
\end{aligned}
$$

Theorem 1. Let the conditions $\mathrm{H} 1$ and $\mathrm{H} 2$ hold. 
Then the system (1)-(4) has at least one periodic solution with initial point $\left(m_{0}, M_{0}\right) \in \Phi_{1}^{c, C, \min }$ and period $T=t_{k_{0}}=t_{k_{0}}\left(m_{0}, M_{0}\right)$.

Proof. For convenience, the proof will be presented in several parts.

Part 1. Let the initial point $\left(m_{0}, M_{0}\right) \in \Phi_{1}^{c, C, \min } \subset G_{1}^{c, C}$. The solution of initial value problem (1)-(5) is defined for $0=t_{0} \leq t \leq T$. In fact, for $0=t_{0} \leq t \leq t_{1}$, the solution of considered problem with variable structure and impulses coincides with the solution of initial value problem (6), (7), (8), where $i=1$ and $\left(m_{01}, M_{01}\right)=\left(m_{0}, M_{0}\right)$ (see equality (9)). From condition $\mathrm{H} 2$, it is seen that the point $\left(m_{1}^{+}, M_{1}^{+}\right) \in \Phi_{2}^{c, C, \min } \subset G_{2}^{c, C}$. For $t_{i-1}<t \leq t_{i}$, the solution of (1)-(5) coincides with the solution of initial value problem (6), (7), (8), where $i=2,3, \ldots, k_{0}$ and the initial point $\left(m_{0 i}, M_{0 i}\right)=\left(m_{i}^{+}, M_{i}^{+}\right) \in \Phi_{i+1}^{c, C, \text { min }} \subset G_{i+1}^{c, C}$. In other words, for $0<t \leq T$, we have

$$
\begin{gathered}
\left(m\left(t ; m_{0}, M_{0}\right), M\left(t ; m_{0}, M_{0}\right)\right) \\
=\left(m_{i}\left(t ; t_{i-1} ; m_{i-1}^{+}, M_{i-1}^{+}\right), M_{i}\left(t ; t_{i-1} ; m_{i-1}^{+}, M_{i-1}^{+}\right)\right), t_{i-1}<t \leq t_{i}, \quad i=1,2, \ldots, k_{0} .
\end{gathered}
$$

Part 2. Consider the mapping $\varphi_{1}: \Phi_{1}^{c, C, \min } \rightarrow \Phi_{1}^{c, C, \max }$, defined as follows

$$
\begin{aligned}
\left(\forall\left(m_{0}^{+}, M_{0}^{+}\right) \in \Phi_{1}^{c, C, \min }\right) \Rightarrow \varphi_{1}\left(m_{0}^{+}, M_{0}^{+}\right)=\left(m_{1}\left(t_{1} ; 0, m_{0}^{+}, M_{0}^{+}\right), M_{1}\left(t_{1} ; 0, m_{0}^{+}, M_{0}^{+}\right)\right) & \\
& =\left(m_{1}\left(t_{1} ; 0, m_{0}^{+}, M_{0}^{+}\right), M_{1}^{00}\right) \in \Phi_{1}^{c, C, \max } .
\end{aligned}
$$

We will show that the mapping $\varphi_{1}$ is continuous on $\Phi_{1}^{c, C, \min }$. For this purpose, it is sufficient to establish that $m_{1}\left(t_{1} ; 0, m_{0}^{+}, M_{0}^{+}\right)$depends continuously on the initial point $\left(m_{0}^{+}, M_{0}^{+}\right)$. Let a point $\left(m_{0}^{*_{+}}, M_{0}^{*+}\right) \in \Phi_{1}^{c, C, \min }$ and a constant $t_{1}^{*}>0$ be such that:

$$
\begin{array}{ll}
- & \left(m_{1}\left(t ; 0, m_{0}^{*_{+}}, M_{0}^{*_{+}}\right), M_{1}\left(t ; 0, m_{0}^{*+}, M_{0}^{*+}\right)\right) \notin \Phi_{1}^{c, C, \max }, 0 \leq t<t_{1}^{*} ; \\
- & \left(m_{1}\left(t_{1}^{*} ; 0, m_{0}^{*_{+}}, M_{0}^{*_{+}}\right), M_{1}\left(t_{1}^{*} ; 0, m_{0}^{*_{+}}, M_{0}^{*_{+}}\right)\right) \in \Phi_{1}^{c, C, \max } .
\end{array}
$$

For concreteness, we assume that $t_{1}^{*} \leq t_{1}$ is fulfilled. The remaining case is considered analogously. According to the property 13 of Remark 2 , it follows that for each sufficiently small constant $\Delta>0$, we have

$$
M_{1}\left(t_{1}-\Delta ; 0, m_{0}^{*_{+}}, M_{0}^{*_{+}}\right)<M_{1}^{00} ; \quad M_{1}\left(t_{1}+\Delta ; 0, m_{0}^{*_{+}}, M_{0}^{*_{+}}\right)>M_{1}^{00} .
$$

From the theorem of continuous dependence (see Theorem 7.1, § 7, Ch. I, [4]), it follows that

$$
\begin{gathered}
\left(\exists \delta_{1}=\text { const }>0\right):\left(\forall\left(m_{0}^{*_{+}}, M_{0}^{*_{+}}\right) \in \Phi_{1}^{c, C, \min }, \|\left(m_{0}^{*_{+}}, M_{0}^{*_{+}}\right)-\left(m_{0}^{+}, M_{0}^{+}\right) \mid<\delta_{1}\right) \\
\Rightarrow M_{1}\left(t_{1}-\Delta ; 0, m_{0}^{*_{+}}, M_{0}^{*_{+}}\right)<M_{1}^{00} ; \quad M_{1}\left(t_{1}+\Delta ; 0, m_{0}^{*_{+}}, M_{0}^{*_{+}}\right)>M_{1}^{00} ; \\
\quad m_{1}\left(t ; 0, m_{0}^{*_{+}}, M_{0}^{*_{+}}\right)>m_{1}^{00}, \quad t_{1}-\Delta \leq t \leq t_{1}+\Delta ; \\
\left|m_{1}\left(t_{1}-\Delta ; 0, m_{0}^{*_{+}}, M_{0}^{*_{+}}\right)-m_{1}\left(t_{1}-\Delta ; 0, m_{0}^{+}, M_{0}^{+}\right)\right|<\Delta ; \\
\left|m_{1}\left(t_{1}+\Delta ; 0, m_{0}^{*_{+}}, M_{0}^{*+}\right)-m_{1}\left(t_{1}+\Delta ; 0, m_{0}^{+}, M_{0}^{+}\right)\right|<\Delta .
\end{gathered}
$$


From (11), taking into account the assumption (more precisely $t_{1}^{*} \leq t_{1}$ ), we reach the following inequalities $t_{1}-\Delta<t_{1}^{*} \leq t_{1}<t_{1}+\Delta$. According to the property 11 of Remark 2, we have:

$$
\begin{aligned}
& \left|m_{1}\left(t_{1} ; 0, m_{0}^{+}, M_{0}^{+}\right)-m_{1}\left(t_{1}-\Delta ; 0, m_{0}^{+}, M_{0}^{+}\right)\right| \leq \int_{t_{1}-\Delta}^{t_{1}}\left|f_{1}^{1}\left(m_{1}\left(\tau ; 0, m_{0}^{+}, M_{0}^{+}\right)\right)\right| d \tau \leq M_{f_{1}^{1}} \Delta ; \\
& \left|m_{1}\left(t_{1}^{*} ; 0, m_{0}^{*+}, M_{0}^{*+}\right)-m_{1}\left(t_{1}-\Delta ; 0, m_{0}^{*+}, M_{0}^{*+}\right)\right| \leq \int_{t_{1}-\Delta}^{t_{1}^{*}}\left|f_{1}^{1}\left(m_{1}\left(\tau ; 0, m_{0}^{*+}, M_{0}^{*+}\right)\right)\right| d \tau \leq M_{f_{1}^{1}} \Delta .
\end{aligned}
$$

From the above two inequalities and (12), we find

$\left|m_{1}\left(t_{1} ; 0, m_{0}^{*_{+}}, M_{0}^{*+}\right)-m_{1}\left(t_{1} ; 0, m_{0}^{+}, M_{0}^{+}\right)\right|$

$$
\begin{aligned}
& \leq\left|m_{1}\left(t_{1}-\Delta ; 0, m_{0}^{*_{+}}, M_{0}^{*_{+}}\right)-m_{1}\left(t_{1}-\Delta ; 0, m_{0}^{+}, M_{0}^{+}\right)\right| \\
& \leq\left|m_{1}\left(t_{1} ; 0, m_{0}^{*_{+}}, M_{0}^{*_{+}}\right)-m_{1}\left(t_{1}-\Delta ; 0, m_{0}^{*_{+}}, M_{0}^{*_{+}}\right)\right| \\
& \quad+\left|m_{1}\left(t_{1} ; 0, m_{0}^{+}, M_{0}^{+}\right)-m_{1}\left(t_{1}-\Delta ; 0, m_{0}^{+}, M_{0}^{+}\right)\right| \\
& \leq\left(1+2 M_{f_{1}^{1}}\right) \Delta .
\end{aligned}
$$

From (10) and (14), we conclude that

$$
\begin{aligned}
& (\forall \varepsilon>0)(\exists \delta=\text { const }>0):\left(\forall\left(m_{0}^{*+}, M_{0}^{*+}\right) \in \Phi_{1}^{c, C, \min },\left\|\left(m_{0}^{*_{+}}, M_{0}^{*+}\right)-\left(m_{0}^{+}, M_{0}^{+}\right)\right\|<\delta\right) \\
& \Rightarrow\left|m_{1}\left(t_{1} ; 0, m_{0}^{*+}, M_{0}^{*+}\right)-m_{1}\left(t_{1} ; 0, m_{0}^{+}, M_{0}^{+}\right)\right|<\varepsilon .
\end{aligned}
$$

Thus, we have proved that the mapping $\varphi_{1}$ is continuous.

Part 3. Consider the mapping $\psi_{1}: \Phi_{1}^{c, C, \max } \rightarrow \Phi_{2}^{c, C, \min }$ defined as follows

$$
\left(\forall\left(m_{1}, M_{1}\right) \in \Phi_{1}^{c, C, \max }\right) \Rightarrow \psi_{1}\left(m_{1}, M_{1}\right)=\left(g_{1}\left(m_{1}, M_{1}\right), M_{2}^{00}\right) \in \Phi_{2}^{c, C, \min } .
$$

According to condition $\mathrm{H} 2$, the mapping $\psi_{1}$ is continuous.

Part 4. Consider the mapping $\varphi_{i}: \Phi_{i}^{c, C, \min } \rightarrow \Phi_{i}^{c, C, \max }, i=2,3, \ldots, k_{0}$, which is defined by means of the equalities

$$
\begin{array}{r}
\left(\forall\left(m_{i-1}^{+}, M_{i-1}^{+}\right) \in \Phi_{i}^{c, C, \min }\right) \Rightarrow \varphi_{i}\left(m_{i-1}^{+}, M_{i-1}^{+}\right)=\left(m_{i}\left(t_{i} ; 0, m_{i-1}^{+}, M_{i-1}^{+}\right), M_{i}\left(t_{i} ; 0, m_{i-1}^{+}, M_{i-1}^{+}\right)\right) \\
=\left(m_{i}\left(t_{i} ; 0, m_{i-1}^{+}, M_{i-1}^{+}\right), M_{i}^{00}\right) \in \Phi_{i}^{c, C, \max } .
\end{array}
$$

Similarly as in Part 2, it can be established that this mapping is continuous on its domain.

Part 5. Let the mapping $\psi_{i}: \Phi_{i}^{c, C, \max } \rightarrow \Phi_{i+1}^{c, C, \min }, i=2,3, \ldots, k_{0}$, be defined as follows

$$
\left(\forall\left(m_{i}, M_{i}\right) \in \Phi_{i}^{c, C, \max }\right) \Rightarrow \psi_{i}\left(m_{i}, M_{i}\right)=\left(g_{i}\left(m_{i}, M_{i}\right), M_{i+1}^{00}\right) \in \Phi_{i+1}^{c, C, \min } .
$$

According to condition $\mathrm{H} 2$, the mapping $\psi_{i}$ is continuous.

Part 6. Let us introduce the mapping $F: \Phi_{1}^{c, C, \min } \rightarrow \Phi_{k_{0}+1}^{c, C, \min }$, where

$$
\left(\forall\left(m_{0}^{+}, M_{0}^{+}\right) \in \Phi_{1}^{c, C, \min }\right)
$$




$$
\Rightarrow F\left(m_{0}^{+}, M_{0}^{+}\right)=\psi_{k_{0}}\left(\varphi_{k_{0}}\left(\psi_{k_{0}-1}\left(\varphi_{k_{0}-1}\left(\ldots\left(\psi_{1}\left(\varphi_{1}\left(m_{0}^{+}, M_{0}^{+}\right)\right)\right) \ldots\right)\right)\right)\right) .
$$

From condition $\mathrm{H} 1$, taking in mind Remark 6 , we find that $\Phi_{k_{0}+1}^{c, C, \min }=\Phi_{1}^{c, C, \text { min }}$, i.e. $F: \Phi_{1}^{c, C, \min } \rightarrow \Phi_{1}^{c, C, \text { min }}$. From previous parts (with numbers 2, 3, 4 and 5) of the proof, it follows that $F \in C\left[\Phi_{1}^{c, C, \min }, \Phi_{1}^{c, C, \min }\right]$. From Remark 4, we have that $\Phi_{1}^{c, C, \text { min }}$ is compact, connected and convex set. Using the Brouwer fixed point theorem, we reach the conclusion that

$$
\left(\exists\left(m_{0}, M_{0}\right) \in \Phi_{1}^{c, C, \min }\right): F\left(m_{0}, M_{0}\right)=\left(m_{0}, M_{0}\right) .
$$

Part 7. Taking into account the definitions of functions $\varphi_{i}$ and $\psi_{i}, i=1,2, \ldots, k_{0}$, we conclude that

$$
F:\left(m_{0}, M_{0}\right) \rightarrow\left(m\left(t_{k_{0}}+0 ; m_{0}, M_{0}\right), M\left(t_{k_{0}}+0 ; m_{0}, M_{0}\right)\right)
$$

and therefore,

$$
\left(m\left(t_{k_{0}}+0 ; m_{0}, M_{0}\right), M\left(t_{k_{0}}+0 ; m_{0}, M_{0}\right)\right)=\left(m_{0}, M_{0}\right) \text {. }
$$

Finally, from conditions $\mathrm{H} 1$ and $\mathrm{H} 2$, we obtain that $\left(m\left(t ; m_{0}, M_{0}\right), M\left(t ; m_{0}, M_{0}\right)\right)$ is a periodic solution of the initial value problem (1)-(5). The period of solution is $T=t_{k_{0}}$.

The theorem is proved.

\section{ACKNOWLEDGEMENTS}

The authors thank to the University of Chemical Technology and Metallurgy, Sofia for the financial support.

\section{REFERENCES}

[1] Chukleva, R., Dishliev, A. and Dishlieva, K., Continuous dependence of the solutions of the differential equations with variable structure and impulses in respect of switching functions, International J. of Applied Science and Technology, 1 (5), (2011), 46-59.

[2] Chukleva, R., Dishliev, A. and Dishlieva, K., Stability of differential equations with variable structure and nonfixed impulsive moments using sequences of Lyapunov's functions, International J. of Differential Equations and Applications, 11 (1), (2012), 57-80.

[3] Coddington, E. and Levinson, N., Theory of ordinary differential equations, McGraw-Hill Book Company, New York, Toronto, London, (1955).

[4] Dishliev, A. and Bainov, D., Continuous dependence of the solution of a system of differential equations of a system of differential equations with impulses on the impulse hypersurfaces, J. of Mathematical Analysis and Applications, 135 (2), (1988), 369-382.

[5] Guo, H. and Chen, L., Time-limited pest control of a Lotka-Volterra model with impulsive harvest, Nonlinear Analysis Real World Applications, 10 (2), (2009), 840-848.

[6] Liu, B., Teng, Z. and Liu, W., Dynamic behaviors of the periodic Lotka-Volterra competing system with impulsive perturbations, Chaos Solitons \& Fractals, 31 (2), (2007), 356-370.

[7] Milev, N. and Bainov, D., Dichotomies for linear impulsive differential equations with variable structure, International J. of Theoretical Physics, 31 (2), (1992), 353-361.

[8] Milev, N., Bainov, D. and Roach, G., Stability of linear systems of differential equations with variable structure and impulse effect, Mathematical Methods in the Applied Sciences, 11 (2), (1989), 271-278.

[9] Nie, L., Teng, Z., Hu, L. and Peng, J., The dynamics of a Lotka-Volterra predator-prey model with state dependent impulsive harvest for predator, BioSystems, 98 (2), (2009), 67-72.

[10] Xia, Y., Positive periodic solutions for a neutral impulsive delayed Lotka-Volterra competition system with the effect of toxic substance, Nonlinear Analysis: Real World Applications, 8 (1), (2007), 204-221.

[11] Yan, J., Zhao A. and Nieto, J., Existence and global attractively of positive periodic solution of periodic single-species impulsive Lotka-Volterra systems, Mathematics and Computer Modeling, 40 (5-6), (2004), 509-518. 


\section{About the Authors}

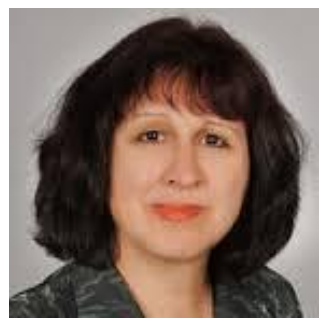

Katya Dishlieva is an associated professor of differential equations at faculty of Applied Mathematics and Informatics, Technical University of Sofia, Bulgaria. Research interests: stabilty theory and periodic solutions of ordinary differential equations with impulses, transition "High school-University", testing and training tests, interdisciplinary connections.

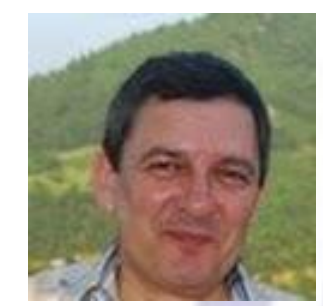

Andrey Antonov is an assistant professor at University of Chemical Technology and Metallurgy, Sofia, Bulgaria. Research interests: dynamical systems, impulsive differential equations, 3D modelling and programming, integral point sets.

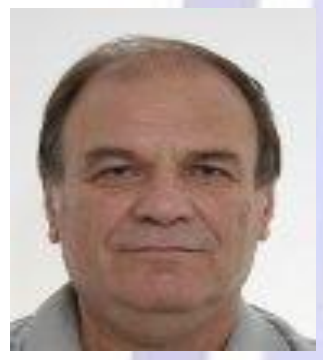

Angel Dishliev is a professor and head of the Department of Mathematics at University of Chemical Technology and Metallurgy, Sofia, Bulgaria. Research interests: qualitative theory of differential equations and applications. 